\title{
Prosthetic Rehabilitation of A Partially Amputated Finger Using A Silicone Prosthesis : A case report
}

\begin{abstract}
:
Loss always has a negative impact on an individualwhich engraves a feeling of incompleteness in many. Loss of anybody part creates an obstruction in normal functioning of body. Mostly the appendages such as arms, fingers etc have very important role in routine life which can restrict the living psychologically, functionally and socially. The goal in rehabilitating a body part should be to create a substitute which is indistinguishable from adjacent and contralateral counterparts. Therefore maxillofacial prosthodontics is not only science but also an art. Present article is a case report of a person with missing finger briefing the fabrication methodology of a custom made silicone finger prosthesis.
\end{abstract}

Keywords: Partial finger amputation, Customized silicone finger prosthesis, Ring attachment.

\section{Introduction:}

Hand is an important organ of our body which acts as a distal function tool facilitating various day to day functions like grasping, touching etc. The five fingers called phalanges forms the hand which makes it the most functionable organ.[1] Complete or partial finger amputations are very often observed in our day to day life. Although injury due to trauma is the most prevailing cause of the amputation but congenital defect and malformations are also encounterd clinically.[2]

Loss of a part or whole of the finger emotionally disturbs the patient and often leads to serious functional deficiencies. Prosthetic rehabilitation of a missing finger is an effective solution which can bring change in life to some extent and can put a great impact on the patient emotionally and physically. It also makes the patient more socially acceptable.[3] Surgical procedures cannot always restore esthetics as efficiently as a prosthesis and also puts a financial burden on the patient. Thus the idea of a finger prosthesis must be such that it should meet the following preconditions. It must aid in grasping, absorbing and transferring the forces to the hand, appear natural and allow expression of gestures.[4,5]

\begin{tabular}{|l|l|}
\hline \multicolumn{3}{|c|}{ Access this article online } \\
\hline \multirow{2}{*}{$\begin{array}{l}\text { Website: } \\
\text { www.ujds.in }\end{array}$} & Quick Response Code \\
\hline $\begin{array}{l}\text { DOI: } \\
\text { https://doi.org/10.21276/ujds.2020.6.3.17 }\end{array}$ & \\
\hline
\end{tabular}

This case report explains the prosthetic rehabilitation of a finger defect using a silicone prosthesis.

\section{Case Report:}

A 28 years old male patient reported to the Department of Prosthodontics, Crown and Bridge with the chief complaint of partial loss of the middle finger of the right hand. In history he disclosed that he lost the finger while working on a mechanical lathe 2 years back. On examination it was evaluated clinically that a residual stump of approximately $2 \mathrm{~cm}$ in height was present with a solitary scar at the base. No abnormality was detected in the surrounding areas. The prosthetic restoration was planned and the patient was educated about the procedure and a signed consent was taken from the patient before

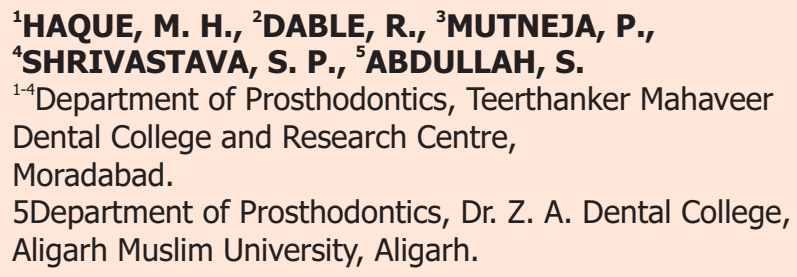

Address for Corresponding : Dr. Merazul Haque Department of Prosthodontics

Teerthanker Mahaveer Dental College and Research

Centre, Moradabad

Email id- merazualhaque13@gmail.com

Received : 5 Nov. 2020, Published : 31 Dec. 2020

How to cite this article: Haque, M. H., Dable, R., Mutneja, P., Shrivastava, S. P., \& Abdullah, S. (2021). The Prosthetic Rehabilitation of a partially amputated finger using a silicon prosthesis: A case report . UNIVERSITY JOURNAL OF DENTAL SCIENCES, 6(3). 
commencing the procedure in order to ensure his willingness and cooperation.[2]

\section{Clinical procedure:}

- After lubricationpatientshand was prepared for making an impression with irreversiblehydrocolloid.

- A plastic bottle was cut and the bottom part was used as a tray to hold the material. Irreversible hydrocolloid material was mixed and poured into the tray. The patient was instructed to insert the hand into the tray while keeping his palm and fingers in a totally relaxed position without stretching from the normal position. The patient was guided to retrieve his hand very cautiously in the same path of positioning. (Figure 1)

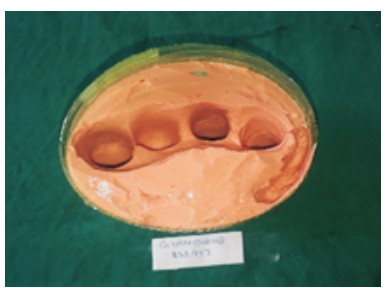

Figure 1: Impression of defected finger.

- The impression was poured with dental stone and was kept over the vibrator toavoid voids. On setting of dental stone the positivereplica of thehand was retrieved and examined for accuracy.(Figure 2)

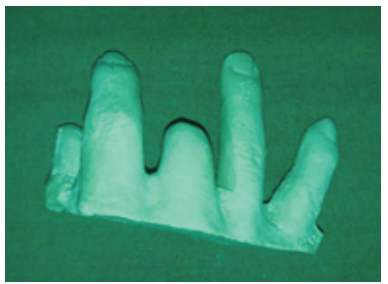

Figure 2: Stone cast obtained from the impression.

- A donor was selected whose fingers were similar in size and shape as that of the patient. Following the same method of impression making an impression of the donors right hand middle finger was made.(Figure 3 )

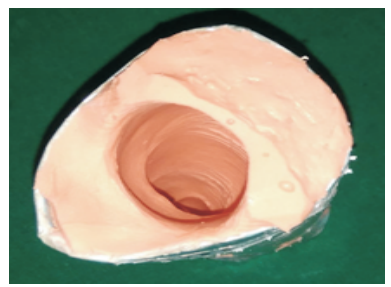

Figure 3: Impression of the donor finger.

Molten modelling waxwas immediately poured into thedonors finger impression by finger gloves technique to duplicate the lostfinger. In order to reduce the weight of the prosthesis the inner surface of the wax pattern was made hollow. (Figure 4)

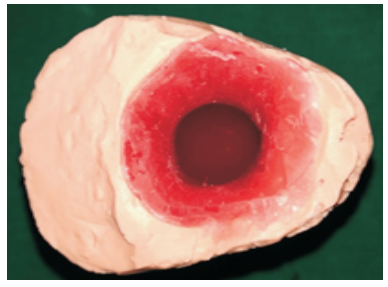

Figure 4: Pouring the modelling wax into the donors impression

The wax pattern was retrieved from the impression. (Figure 5)

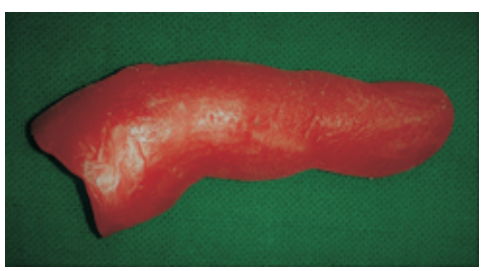

Figure 5: Wax pattern

This wax pattern was modified to adapt and remove from the model with outdistortion. (Figure 6)

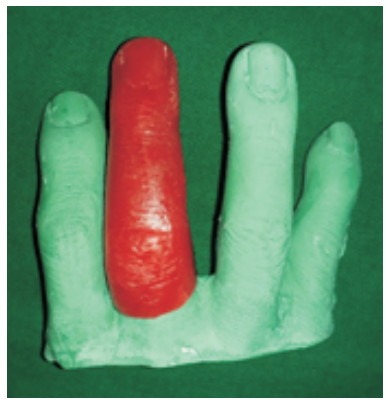

Figure 6: Wax pattern adaptation on the stone cast. The patient was recalled for wax pattern trial. (Figure 7)

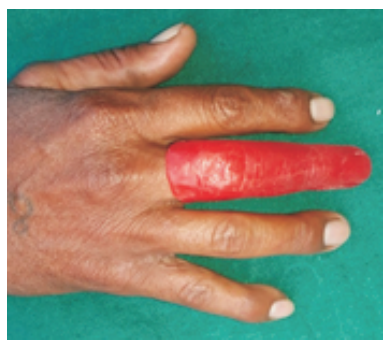

Figure 7: Wax pattern trial.

The stump was prepared by beading of the stump on the ventral surface.(Figure 8) 


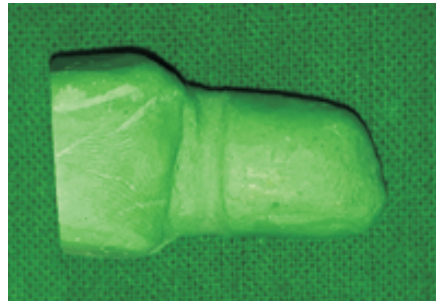

Figure 8:Stumpof defected finger.

Flasking of the stump with the wax pattern was done in such a way that the dorsal and the ventral aspects were flasked separately in the two pours. This was done to enhance the precision while shade matching.(Figure 9). The dewaxing was done in the conventional way andthemould was allowed to cooldown. (Figure 10)

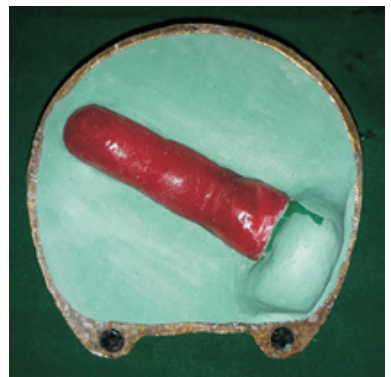

Figure 9: Flasking of wax pattern.

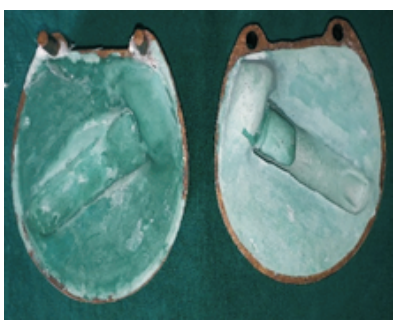

Figure 10: Dewaxing of wax pattern.

Silicone material was planned to be used for fabricating this prosthesis. Shade matching was done in front ofthe patient to obtain a similar characterization of thepalmer and dorsal surfaces intrinsic colourswere mixed in the silicon. Shade matching was done separately for the dorsal and ventral surfaces. (Figure 11)

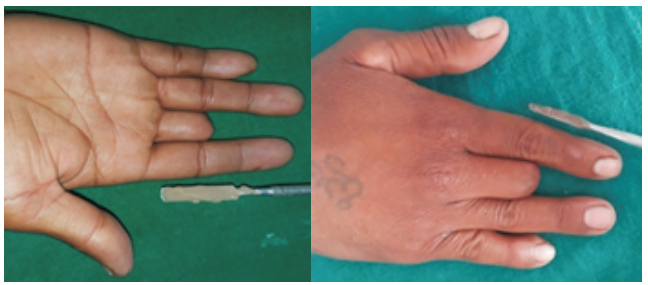

Figure 11: Shade matching.
The material was mixed separately for the dorsal and the ventral surfaces and were packed in their respective segments of the flask. It wasleft overnight for bench curingat room temperature to allow complete polymerization. The flasks were deflaskedand final prosthesis was retrieved. Excess material was cut using scissor. Sand papering was done from coarse to finer gritsto get the final finish.(Figure 12)
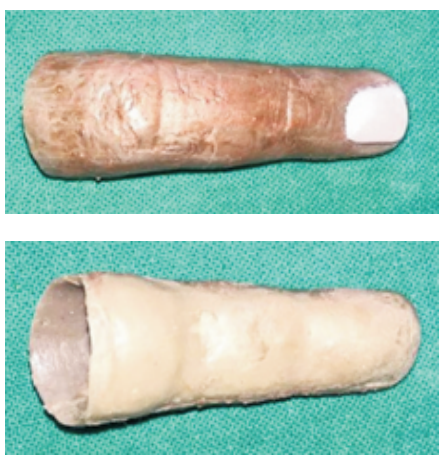

Figure 12: Final finger prosthesis.

To enhance retention in the final prosthesis a ring attachment was used. (Figure:13)

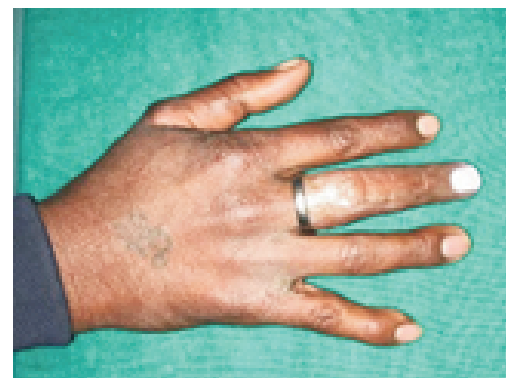

Figure 13: Final prosthesis

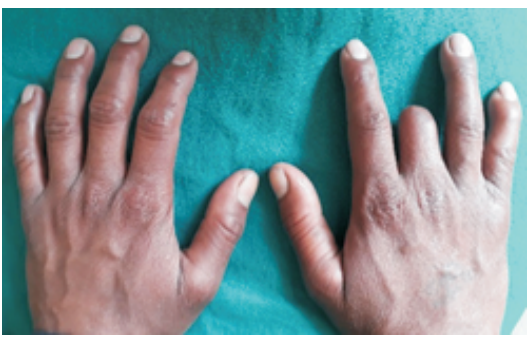

Figure 14 a: pre-prosthetic

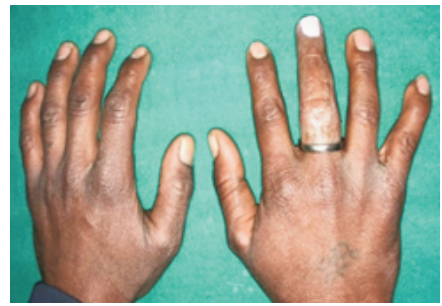

Figure 14 b: post-prosthetic 


\section{Discussion:}

Prosthetic rehabilitation of missing part of body can be of great help when surgical reconstruction is not possible. Along with function and esthetics it provides a great amount of psychological help. It improves a quality of life of patient and motivates the patient to lead a comparatively normal professional and social life.[6]

A successful finger prosthesis is the one that resembles the digit of the contralateral hand. There are various materials in current maxillofacial prosthesis. The material which is to be used should be strong and repairable, comfortable to clean, does not get stained with extrinsic stains and be biocompatible. Thus silicone and acrylic resin are the most routinely used materials for the prosthesis.[1]

Appropriate prosthesis are routinely used to rehabilitate phalangeal amputations to normal functionality.7When it comes to the acceptability of the prosthesis customized silicone prostheses are frequently selected because it is comfortable, durable and is stain resistant. Thus silicone is better when compared to the other maxillofacial materials. Water and soap are used to remove all the stains from a silicone prosthesis. Thesilicone gel makes the hydration of the stratum corneum of immature hypertropic scars better.[8]

There are numerous methods to achieve the retention of the prosthesis like ring - attachments, medical grade adhesive or implants. The choice of selection of the mode of retention depends entirely on the length and form of the residual stump. For this case vaccum retention was achieved due to the snug fit of the prosthesis by beading of the stump. Along with this to mask the margin of the prosthesis and make it more esthetically pleasant a ring was attached which also provided additional retention. $[6,8]$

Although the prosthetic devices does not reconstruct the hand in cases of injury or amputation but it does assist in recovering the basic functionality of the hand.

\section{Conclusion:}

Prosthetically rehabilitating a defect may not completely restore the function but it does help an individual to overcome the psychological trauma and lead a healthy life. for this patient prosthetic rehabilitation of the partially amputated middle finger was done using custom made silicone finger prosthesis which motivated him to overcome the post traumatic stresses.

\section{References:}

1. Lotwani V, Jaswi A. Prosthetic Rehabilitation of a lost finger using heat cure acrylic resin- A case report. JOADMS 2015;1(3):76-80.

2. RichaVashisht, D. R. Prithviraj, HarleenKaur, "Finger prosthesis using silicone elastomer: A novel methodology," Int J ContempDent Med Rev, vol.2015.

3. Miglani DC, Drane JB. Maxillofacial prosthesis and its role as a healing art. J Prosthet Dent 1959;9:159-68.

4. Pereira BP, Kour AK, Leow EL, Pho RW. Benefits and use of digital prostheses. J Hand Surg Am 1996;21:222-8.

5. Pillet J. Esthetic hand prostheses. J Hand Surg Am 1983;8:778-81.

6. FernandezTeny, Harshakumar K, Ravichandran R. Prosthetic Rehabilitation of a partially amputed finger using a silicone prosthesis. IJOPRD 2016;6(1):10-13

7. Larcher S, Espen D: Post-acute management of fractures of the proximal interphalangeal joint with metal prosthesis: first experience. Handchir Mikrochir Plast Chir 2007;39:263-266.

8. KairaLaxman Singh, HandaMohit, DabralEsha, Gupta KK. Silicon Finger Prosthesis for A Partial FingerAmputation: A Case Report. AJPP 2014;1(3):105111 